\title{
Improved Non-Repudiable Threshold Proxy Signature Scheme with Known Signers
}

\author{
Chwei-Shyong TSAI ${ }^{b}$, Shiang-Feng TZENG ${ }^{a}$, Min-Shiang $\mathrm{HWANG}^{a, c}$ \\ ${ }^{a}$ Department of Information Management, Chaoyang University of Technology \\ 168, Gifeng E. Rd., Wufeng, Taichung County, Taiwan 413, ROC \\ e-mail: mshwang@mail.cyut.edu.tw \\ ${ }^{b}$ Department of Information Management, National Taichung Institute of Technology \\ 129 Sec. 3, San-min Rd., Taichung, Taiwan 404, ROC \\ ${ }^{c}$ Department of Management Information Systems, National Chung Hsing University \\ 250, Kuo Kuang Road, Taichung, Taiwan 402, ROC
}

Received: March 2003

\begin{abstract}
In 2001, Hsu et al. proposed a non-repudiable threshold proxy signature with known signers. In their scheme, the proxy group cannot deny having signed the proxy signature if they did. However, Hsu et al.'s scheme is vulnerable to some attacks. A malicious original signer or malicious proxy signer can impersonate some other proxy signers to generate proxy signatures. In this article, we shall present our cryptanalysis of the Hsu et al.'s scheme. After that, we shall propose a new threshold proxy signature that can overcome the weaknesses.
\end{abstract}

Key words: digital signature, proxy signature, threshold proxy signature.

\section{Introduction}

The concept of a proxy signature (Mambo et al., 1996a; Mambo et al., 1996b) was first introduced in 1996. The proxy signature scheme allows the original signer to delegate her/his signing capability to a designated person, called a proxy signer. The proxy signer generates the proxy signature on a message on behalf of the original signer. After that, any verifier can check the validity of the proxy signature and can make sure of the original signer's agreement on the signed message.

Following the development of the proxy signature scheme, some threshold proxy signature schemes have been proposed and widely studied (Hsu et al., 2001; Hwang et al., 2000; Hwang et al., 2002b; Kim et al., 1997; Sun, 1999; Zhang, 1997). In a $(t, n)$ threshold proxy signature scheme, which is a variant of the proxy signature scheme, the proxy signature key is shared among a group of $n$ proxy signers delegated by the original signer. Any $t$ or more proxy signers can cooperatively sign messages on behalf of the original signer.

Sun (1999) proposed an efficient non-repudiable threshold proxy signature scheme with known signers based on Kim's scheme (Kim et al., 1997). Sun's scheme is more 
efficient than other threshold proxy signature schemes and has the non-repudiable property. The main advantage of Sun's scheme is that the verifier is able to identify the actual signers in the proxy group. However, Sun's scheme is vulnerable to the collusion attack (Hwang et al., 2000) and the conspiracy attack (Hsu et al., 2001).

Hsu et al. (2001) proposed a new and efficient non-repudiable proxy signature scheme that could withstand the above attacks. Furthermore, it also outperformed Sun's scheme in computational complexity and communication cost. However, we will show that Hsu et al.'s scheme is vulnerable to the public key substitution and the insider forgery attacks in this article. A malicious original signer can impersonate $t$ or more legal proxy signers to generate proxy signatures. Besides, the malicious proxy signer can impersonate $t-1$ or more legal proxy signers to generate proxy signatures. Moreover, the real proxy signers cannot deny having signed the proxy signature before though they did not.

In this article, we shall show the weaknesses of Hsu et al.'s scheme and remedy the problems. In the next section, we shall review Hsu et al.'s scheme (the Hsu-Wu-Wu scheme). In Section 3, we shall show that the Hsu-Wu-Wu scheme is vulnerable to the public key substitution attack (Sun, 2000) and the insider forgery attack (Li et al., 2000). In Section 4, our improved scheme and the security analysis of the improved scheme will be proposed and presented. Finally, the concluding remarks will be in the last section.

\section{Review of the Hsu-Wu-Wu Scheme}

The scheme includes four phases: secret share generation, proxy share generation, proxy signature generation, and proxy signature verification. There exists a system authority (SA) whose tasks are to initialize the system and to manage the public directory. In the secret share generation phase, initially, SA selects and publishes the following parameters:

- $p$ : a large prime,

- $q$ : a large prime factor of $p-1$,

- $g$ : a generator in $G F(p)$ of order $q$,

- $h(\cdot)$ : a one-way hash function,

- $M_{w}$ : a warrant which records the identities of the original signer and the proxy signers of the proxy group, parameters $t$ and $n$, the valid delegation time, etc.,

- ASID: (Actual Signers' $I D$ ) the identities of the actual signers.

Each user $U_{i}$, with the public identifier $v_{i} \in Z_{q}$, owns a private key $x_{i} \in Z_{q}^{*}$ and a public key $y_{i}=g^{x_{i}} \bmod p$ which is certified by a certificate authority (CA). Let $U_{O}$ be the original signer and $G_{P}=\left\{U_{P_{1}}, U_{P_{2}}, \cdots, U_{P_{n}}\right\}$ be the proxy group of $n$ proxy signers. 


\subsection{Secret Share Generation Phase}

SA selects the group private key $X_{G}$ and calculates the group public key $Y_{G}=g^{X_{G}} \bmod$ $p$ which is certified by CA. Then, SA randomly creates a $(t-1)$-degree polynomial as

$$
f(v)=X_{G}+a_{1} v+a_{2} v^{2}+\cdots+a_{t-1} v^{t-1} \bmod q,
$$

where the random integers $a_{i} \in Z_{q}(i=1,2, \cdots, t-1)$.

For each $U_{P_{i}} \in G_{P}$, SA calculates the secret share $\gamma_{i}=f\left(v_{i}\right)$ and the corresponding public information $\tau_{i}=g^{\gamma_{i}} \bmod p$, where $v_{i}$ is the public identifier for $U_{P_{i}}$. Then, SA separately sends $\gamma_{i}$ to $U_{P_{i}}$ via a secure channel and publishes all $\tau_{i}$ 's.

\subsection{Proxy Share Generation Phase}

$U_{O}$ performs the following steps to delegate the signing capability to $G_{P}$.

1. Select a random number $k \in Z_{q}^{*}$ and calculate $K=g^{k} \bmod p$.

2. Calculate the proxy signature key as $\sigma=k+x_{O} h\left(M_{w} \| K\right) \bmod q$, where "\|" denotes the concatenation operator.

3. Generate a polynomial $f_{O}(v)=\sigma+b_{1} v+b_{2} v^{2}+\cdots+b_{t-1} v^{t-1} \bmod q$, where the random numbers $b_{j} \in Z_{q}(j=1,2, \cdots, t-1)$.

4. Publish $B_{j}=g^{b_{j}} \bmod p$, for $j=1,2, \cdots, t-1$.

5. Send $\sigma_{i}=f_{O}\left(v_{i}\right)$ to $U_{P_{i}} \in G_{P}$ via a secure channel.

6. Broadcast $\left(M_{w}, K\right)$.

Receiving $\sigma_{i}$, each $U_{P_{i}} \in G_{P}$ can validate it by checking the following equation

$$
g^{\sigma_{i}} \stackrel{?}{=} y_{O}^{h\left(M_{w} \| K\right)} K\left(\prod_{j=1}^{t-1} B_{j}^{v_{i}^{j}}\right) \bmod p .
$$

If it holds, $U_{P_{i}}$ calculates $\sigma_{i}^{\prime}=\sigma_{i}+\gamma_{i} h\left(M_{w} \| K\right) \bmod q$ as her/his proxy share.

\subsection{Proxy Signature Generation Phase}

Given a message $M$, any $t$ or more proxy signers of $G_{P}$ will be the proxies for $U_{O}$ to sign $M$ in this phase. Without loss of generality, let $D_{P}=\left\{U_{P_{1}}, U_{P_{2}}, \cdots, U_{P_{t}}\right\}$ be the actual proxy signers. $D_{P}$ as a group performs the following steps to generate the proxy signature.

1. Each $U_{P_{i}}$ selects a random number $k_{i} \in Z_{q}^{*}$ and then broadcasts $r_{i}=g^{k_{i}} \bmod p$.

2. Upon receiving all $r_{j}$ 's $(j=1,2, \cdots, t ; j \neq i)$, each $U_{P_{i}}$ calculates

$$
\begin{aligned}
R & =\prod_{j=1}^{t} r_{j} \bmod p, \\
s_{i} & =k_{i} R+\left(L_{i} \sigma_{i}^{\prime}+x_{P_{i}}\right) h(R\|A S I D\| M) \bmod q,
\end{aligned}
$$


where $L_{i}=\prod_{j=1, j \neq i}^{t}\left(-v_{j}\right)\left(v_{i}-v_{j}\right)^{-1} \bmod q$. Here, $s_{i}$ is the individual proxy signature which is sent to the designated clerk.

3. Upon receiving $s_{i}$, the designated clerk validates it by checking

$$
g^{s_{i}} \stackrel{?}{=} r_{i}^{R}\left(\left(\left(y_{O} \tau_{i}\right)^{h\left(M_{w} \| K\right)}\left(\prod_{j=1}^{t-1} B_{j}^{v_{i}^{j}}\right) K\right)^{L_{i}} y_{P_{i}}\right)^{h(R\|A S I D\| M)} \bmod p
$$

If it holds, $\left(r_{i}, s_{i}\right)$ is the valid individual proxy signature of $M$. If all the individual proxy signatures of $M$ are valid, the clerk calculates

$$
S=\sum_{j=1}^{t} s_{j} \bmod q
$$

The proxy signature of $M$ is $\left(R, S, K, M_{w}, A S I D\right)$.

\subsection{Proxy Signature Verification Phase}

Receiving the proxy signature $\left(R, S, K, M_{w}, A S I D\right)$ of $M$, any verifier can verify the validity of the proxy signature and identify the actual signers. The steps of this phase are described as follows:

1. According to $M_{w}$ and $A S I D$, the verifier can identify the original signer and the proxy signers, and obtain the necessary public keys from the CA. In addition, she/he can identify the actual proxy signers, too.

2. The verifier validates the proxy signature by checking

$$
g^{S} \stackrel{?}{=} R^{R}\left(K\left(y_{O} Y_{G}\right)^{h\left(M_{w} \| K\right)} \prod_{i=1}^{t} y_{P_{i}}\right)^{h(R\|A S I D\| M)} \bmod p .
$$

If it holds, the proxy signature $\left(R, S, K, M_{w}, A S I D\right)$ for $M$ is valid.

\section{The Weaknesses of the Hsu-Wu-Wu Scheme}

In this section, we show that the $\mathrm{Hsu}-\mathrm{Wu}-\mathrm{Wu}$ scheme is vulnerable to the public key substitution attack and the insider forgery attack. A malicious original signer $\left(U_{O}\right)$ or a malicious proxy signer $\left(U_{P_{k}}\right)$ can forge the valid proxy signature without the other signers' private keys.

\subsection{Public Key Substitution Attack}

In this subsection, we will show the Hsu-Wu-Wu scheme is vulnerable to the public key substitution attack. Suppose the malicious original signer, without any private keys of the other proxy signers, attempts to forge a valid proxy signature for a message. The steps of this attack are as follows: 
1. $U_{O}$ randomly selects a private key $x_{O} \in Z_{q}^{*}$.

2. $U_{O}$ waits until she/he obtains any $t$ or more proxy signers' $y_{P_{i}}$. Then, instead of broadcasting $y_{O}=g^{x_{O}} \bmod p$, she/he calculates

$$
y_{O}^{\prime}=g^{x_{O}}\left(Y_{G}\right)^{-1} \prod_{i=1}^{t} y_{P_{i}}^{-h\left(M_{w} \| K\right)^{-1}} \bmod p,
$$

and reveals the value $y_{O}^{\prime}$ as her/his public key.

3. $U_{O}$ selects two random numbers $k$ and $r$, and calculates $K$ and $R$ as follows:

$$
\begin{aligned}
K & =g^{k} \bmod p, \\
R & =g^{r} \bmod p .
\end{aligned}
$$

4. $U_{O}$ chooses a message $M$ at will and calculates $S$ as

$$
S=r R+\left(k+x_{O} h\left(M_{w} \| K\right)\right) h(R\|A S I D\| M) \bmod q .
$$

Then, the proxy signature for $M$ is $\left(R, S, K, M_{w}, A P S I D\right)$.

Theorem 1. A forged proxy signature generated by $U_{O}$ according to the above steps, namely $\left(R, S, K, M_{w}, A S I D\right)$, is a valid proxy signature for the message $M$.

Proof. On receipt of ( $\left.R, S, K, M_{w}, A S I D\right)$, the verifier checks the validity of the proxy signature of the message $M$ through (3) as follows.

$$
\begin{aligned}
g^{S} & =R^{R}\left(K\left(y_{O} Y_{G}\right)^{h\left(M_{w} \| K\right)} \prod_{i=1}^{t} y_{P_{i}}\right)^{h(R\|A S I D\| M)}, \\
& =R^{R}\left(K\left(g^{x_{O}}\left(Y_{G}\right)^{-1} \prod_{i=1}^{t} y_{P_{i}}^{-h\left(M_{w} \| K\right)^{-1}} Y_{G}\right)^{h\left(M_{w} \| K\right)} \prod_{i=1}^{t} y_{P_{i}}\right)^{h(R\|A S I D\| M)} \\
& =R^{R}\left(K g^{x_{O} h\left(M_{w} \| K\right)}\right)^{h(R\|A S I D\| M)} \\
& =g^{r R+\left(k+x_{O} h\left(M_{w} \| K\right)\right) h(R\|A S I D\| M)} \bmod p .
\end{aligned}
$$

The above equation holds, and the forged proxy signature $\left(R, S, K, M_{w}, A S I D\right)$ is taken for the valid proxy signature for message $M$.

\subsection{Insider Forgery Attack}

In this subsection, we will show the Hsu-Wu-Wu scheme is vulnerable to the insider forgery attack feasible. Suppose a malicious proxy signer $U_{P_{k}}$, without any private key of the other proxy signers, attempts to forge a valid proxy signature for an arbitrary message. The attacker can take the steps as follows:

1. $U_{P_{k}}$ randomly chooses a private key $x_{P_{k}} \in Z_{q}^{*}$. 
2. $U_{P_{k}}$ waits until she/he obtains any $t-1$ or more proxy signers' $y_{P_{i}}$. Then, instead of broadcasting $y_{P_{k}}=g^{x_{P_{k}}} \bmod p$, she/he calculates

$$
y_{P_{k}}^{\prime}=g^{x_{P_{k}}}\left(K\left(y_{O} Y_{G}\right)^{h\left(M_{w} \| K\right)} \prod_{i=1}^{t-1} y_{P_{i}}\right)^{-1} \bmod p,
$$

and reveals the quantity $y_{P_{k}}^{\prime}$ as her/his public key.

3. $U_{P_{k}}$ chooses a random number $r$ and calculates $R$ as follows:

$$
R=g^{r} \bmod p .
$$

4. $U_{P_{k}}$ chooses an arbitrary message $M$ and calculates $S$ as

$$
S=r R+x_{P_{k}} h(R\|A S I D\| M) \bmod q .
$$

Then, the proxy signature for $M$ is $\left(R, S, K, M_{w}, A S I D\right)$.

Theorem 2. A forged proxy signature generated by $U_{P_{k}}$ according to the above steps, namely $\left(R, S, K, M_{w}\right.$, ASID), is a valid proxy signature for the message $M$.

Proof. On receipt of ( $\left.R, S, K, M_{w}, A S I D\right)$, the verifier checks the validity of the proxy signature of the message $M$ through (3) as follows.

$$
\begin{aligned}
g^{S} & =R^{R}\left(K\left(y_{O} Y_{G}\right)^{h\left(M_{w} \| K\right)} \prod_{i=1}^{t} y_{P_{i}}\right)^{h(R\|A S I D\| M)}, \\
& =R^{R}\left(K\left(y_{O} Y_{G}\right)^{h\left(m_{w} \| K\right)} g^{x_{P_{k}}}\left(K\left(y_{O} Y_{G}\right)^{h\left(M_{w} \| K\right)} \prod_{i=1}^{t-1} y_{P_{i}}\right)^{-1} \prod_{i=1}^{t-1} y_{P_{i}}\right) h(R\|A S I D\| M), \\
& =R^{R} g^{x_{P_{k}} h(R\|A S I D\| M)} \\
& =g^{r R+x_{P_{k}} h(R\|A S I D\| M)} \bmod p .
\end{aligned}
$$

The above equation holds, and the forged proxy signature $\left(R, S, K, M_{w}, A S I D\right)$ is taken for the valid proxy signature for the message $M$.

\section{Improvement and Cryptanalysis}

In this section, we shall modify the Hsu-Wu-Wu scheme to remedy the weaknesses described previously.

\subsection{The Improved Scheme}

In the Hsu-Wu-Wu scheme, the proxy signature can be forged by a malicious original signer or malicious proxy singer. To remedy this weakness, we have modified the Hsu$\mathrm{Wu}-\mathrm{Wu}$ scheme, and the revised scheme is as follows. 
In the proxy share generation phase, we replace $\sigma$ with

$$
\sigma=k+x_{O} y_{O} h\left(M_{w} \| K\right) \bmod q .
$$

Therefore, (1) becomes as follows.

$$
g^{\sigma_{i}} \stackrel{?}{=} y_{O}^{y_{O} h\left(M_{w} \| K\right)} K\left(\prod_{j=1}^{t-1} B_{j}^{v_{i}^{j}}\right) \bmod p .
$$

The other steps of the proxy share generation phase are the same as those of the HsuWu-Wu scheme.

In the proxy signature generation phase, we replace $s_{i}$ with

$$
s_{i}=k_{i} R+\left(L_{i} \sigma_{i}^{\prime}+x_{P_{i}} y_{P_{i}}\right) h(R\|A S I D\| M) \bmod q .
$$

The proxy signer $U_{P_{i}}$ calculates $s_{i}$ from the above equation and sends $s_{i}$ to the designated clerk. The designated clerk can then verify the validity of $s_{i}$ by the following equation:

$$
g^{s_{i}} \stackrel{?}{=} r_{i}^{R}\left(\left(\left(y_{O}^{y_{O}} \tau_{i}\right)^{h\left(M_{w} \| K\right)}\left(\prod_{j=1}^{t-1} B_{j}^{v_{i}^{j}}\right) K\right)^{L_{i}} y_{P_{i}}^{y_{P_{i}}}\right)^{h(R\|A S I D\| M)} \bmod p,
$$

where $L_{i}=\prod_{j=1, j \neq i}^{t}\left(-v_{j}\right)\left(v_{i}-v_{j}\right)^{-1} \bmod q$. Then, the designated clerk calculates $S$ from (2), and the proxy signature on message $M$ is $\left(R, S, K, M_{w}, A S I D\right)$.

Finally, the verifier checks the validity of the proxy signature and identifies the actual proxy signers from the proxy group by checking the following equation:

$$
g^{S} \stackrel{?}{=} R^{R}\left(K\left(y_{O}^{y_{O}} Y_{G}\right)^{h\left(M_{w} \| K\right)} \prod_{i=1}^{t} y_{P_{i}}^{y_{P_{i}}}\right)^{h(R\|A S I D\| M)} \bmod p .
$$

If it holds, the verifier can make sure of the validity of the proxy signature and identify the actual signers. Furthermore, the revised scheme can withstand the public key substitution attack and the insider forgery attack. Neither, the malicious original signer nor anyone malicious proxy signer can forge the proxy signatures.

\subsection{Security Analysis of the Improved Scheme}

The security of the improved scheme is examined as follows. As with the Hsu-WuWu scheme, the level of security is quite desirable. The difference, however, is that our scheme can withstand the public key substitution attack and the insider forgery attack.

Attack 1: Consider the public key substitution attack. The malicious original signer $U_{O}$ tries to impersonate any $t$ or more proxy signers in $G_{P}$ and to forge their proxy signature without the agreement of these proxy signers. 
Analysis of attack 1: $U_{O}$ has to change her/his public key after the public keys of the $t$ or more proxy signers have been determined. Assume $U_{O}$ waits until she/he receives any $t$ proxy signers' public keys $y_{P_{i}}$. She/He substitutes her/his public key $y_{O}$.

Assume $U_{O}$ selects a random number $x_{O}$ as her/his private key. Then, $U_{O}$ has to make her/his public key $y_{O}^{\prime}$ in satisfying the following equation:

$$
y_{O}^{\prime y_{O}^{\prime}}=g^{x_{O}}\left(Y_{G}\right)^{-1} \prod_{i=1}^{t} y_{P_{i}}^{-h\left(M_{w} \| K\right)^{-1}} \bmod p .
$$

In the above equation, suppose $U_{O}$ determines the value $x_{O}$ first. She/He has to obtain the value $y_{O}^{\prime}$ by solving the difficult problem. On the other hand, suppose $U_{O}$ wants to fix $y_{O}^{\prime}$, she/he has to solve the discrete logarithms (ElGamal, 1985; Hwang et al., 2002a; Hwang et al., 2001; Lee et al., 2002) to find her/his private key $x_{O}$. Therefore, the malicious original signer cannot successfully forge any proxy signature for any message by launching the public key substitution attack.

Attack 2: Consider the insider forgery attack. Suppose a malicious proxy signer $U_{P_{k}}$ tries to impersonate any $t-1$ or more of the other proxy signers in $G_{P}$ and to forge the proxy signature without the agreement of these proxy signers.

Analysis of attack 2: Similarly, $G_{P}$ can also launch the insider forgery attack. Without losing generality, suppose that the malicious proxy signer $U_{P_{k}}$ wants to update her/his public key $y_{P_{k}}$. Assume $U_{P_{k}}$ waits until she/he obtains any $t-1$ proxy signers' public keys $y_{P_{i}}$. She/He changes her/his public key $y_{P_{k}} . U_{P_{k}}$ chooses a random number $x_{P_{k}}$ and makes her/his public key $y_{P_{k}}^{\prime}$ as follows:

$$
y_{P_{k}}^{\prime y_{P_{k}}^{\prime}}=g^{x_{P_{k}}}\left(K\left(y_{O} Y_{G}\right)^{h\left(M_{w} \| K\right)} \prod_{i=1}^{t-1} y_{P_{i}}\right)^{-1} \bmod p .
$$

However, $U_{P_{k}}$ cannot create a valid proxy signature. From the above equation, assume the value of $x_{P_{k}}$ is determined first. Then, it is an extremely difficult thing to find a $y_{P_{k}}^{\prime}$ satisfying the equation. On the other hand, if $U_{P_{k}}$ determines the integer $y_{P_{k}}^{\prime}$ first, she/he has to solve the discrete logarithms to find the value of $x_{P_{k}}$. Thus, the insider forgery attack here will not work.

\section{Conclusions}

In this article, we have presented the public key substitution attack and the insider forgery attack on the Hsu-Wu-Wu scheme. In these attacks, the malicious original signer or any malicious insider proxy signer in the proxy group, without any private keys of other proxy signers, can forge a valid proxy signature for any message. We have also proposed a secure improved scheme to remedy the weaknesses of the Hsu-Wu-Wu scheme. In our new scheme, neither the original signer nor any malicious proxy signer can forge the legal proxy signature. 


\section{Acknowledgements}

The authors wish to thank many anonymous referees for their suggestions to improve this paper. Part of this research was supported by the National Science Council, Taiwan, ROC, under contract no. NSC91-2213-E-324-003.

\section{References}

ElGamal, T. (1985). A public-key cryptosystem and a signature scheme based on discrete logarithms. IEEE Transactions on Information Theory, 1(5), 469-472.

Hsu, C.L., T.S. Wu and T.C. Wu (2001). New nonrepudiable threshold proxy signature scheme with known signers. The Journal of Systems and Software, 58(5), 119-124.

Hwang, M.S., I.C. Lin and E.J.L. Lu (2000). A secure nonrepudiable threshold proxy signature scheme with known signers. Informatica, 11(2), 137-144.

Hwang, M.S., C.C. Chang and K.F. Hwang (2002a). An ElGamal-like cryptosystem for enciphering large messages. IEEE Transactions on Knowledge and Data Engineering, 14(2), 445-446.

Hwang, M.S., C.C. Lee and E.J.L. Lu (2001). Cryptanalysis of the batch verifying multiple DSA-type digital signatures. Pakistan Journal of Applied Sciences, 1(3), 287-288.

Hwang, M. S., E. J. L. Lu and Iuon-Chang Lin (2002b). A practical $(t, n)$ threshold proxy signature scheme based on the RSA cryptosystem. IEEE Transactions on Knowledge and Data Engineering (to appear).

Kim, S., S. Park and D. Won (1997). Proxy signatures, revisited. In Proc. of ICICS'97, LNCS, 1334. pp. 223232.

Lee, C.C., M.S. Hwang and L.H. Li (2002). A new key authentication scheme based on discrete logarithms. Applied Mathematics and Computation (to appear).

Li, Z.C., L.C.K. Hui, K.P. Chow, C.F. Chong, H.H. Tsang and H.W. Chan (2000). Cryptanalysis of Harn digital multisignature scheme with distinguished signing authorities. Electronics Letters, 36(4), 314-315.

Mambo, M., K. Usuda and E. Okamoto (1996a). Proxy signatures: Delegation of the power to sign message. IEICE Trans. Fundamentals, E79-A, 1338-1353.

Mambo, M., K. Usuda and E. Okamoto (1996b). Proxy signatures for delegating signing operation. In Proc. Third ACM Conf. on Computer and Communications Security. pp. 48-57.

Sun, H.M. (1999). An efficient nonrepudiable threshold proxy signature scheme with known signers. Computer Communications, 22(8), 717-722.

Sun, H.M. (2000). On proxy (multi-) signature schemes. In 2000 International Computer Symposium, Chiayi, Taiwan. pp. 65-72.

Zhang, K. (1997). Threshold proxy signature schemes. In 1997 Information Security Workshop. pp. 191-197.

C.-S. Tsai received the BS degree in applied mathematics in 1984 from National Chung Hsing University, Taichung, Taiwan. He received the MS degree in computer science and electronic engineer in 1986 from National Center University, Chungli, Taiwan. He received the $\mathrm{PhD}$ degree in computer science and information engineering in 2002 from National Chung Cheng University, Chiayi, Taiwan. Since August 2002, he has been an associate professor of the Department of Information Management at National Taichung Institute of Technology, Taichung, Taiwan. His research interests include image authentication, information hiding, and cryptography.

S.-F. Tzeng received the BS degree in information management from Chaoyang University of Technology (CYUT), Taichung, Taiwan, Republic of China, in 2001. He is currently pursuing his MS degree in information management from CYUT. His current research interests include applied cryptography and data security. 
M.-S. Hwang received the BS in electronic engineering from National Taipei Institute of Technology, Taipei, Taiwan, Republic of China, in 1980; the MS in industrial engineering from National Tsing Hua University, Taiwan, in 1988; and the $\mathrm{PhD}$ in computer and information science from National Chiao Tung University, Taiwan, in 1995. He also studied applied mathematics at National Cheng Kung University, Taiwan, 1984-1986. Dr. Hwang passed the National Higher Examination in field "Electronic Engineer" in 1988. He also passed the National Telecommunication Special Examination in field "Information Engineering", qualified as advanced technician the first class in 1990. From 1988 to 1991, he was the leader of the Computer Center at Telecommunication Laboratories (TL), Ministry of Transportation and Communications, ROC. He was also a project leader for research in computer security at TL in July 1990. He obtained the 1997, 1998, and 1999 Distinguished Research Awards of the National Science Council of the Republic of China. He is currently a professor and chairman of the Department of Information Management, Chaoyang University of Technology, Taiwan, ROC. He is a member of IEEE, ACM, and Chinese Information Security Association. His current research interests include database and data security, cryptography, image compression, and mobile communications.

\title{
Pagerinta neatsisakomo slenkstinio igaliotojo parašo schema su žinomais parašo pateikẻjais
}

\author{
Chwei-Shyong TSAI, Shiang-Feng TZENG, Min-Shiang HWANG
}

$2001 \mathrm{~m}$. Hsu et al. pasiūlè neatsisakomą slenkstini igaliotajị parašą su žinomu parašo pateikejju. Šių autoriu schemoje igaliotiniu grupé negali atsisakyti savo parašo. Deja, Hsu et al. schema yra nesaugi kai kuriu isilaužimų atžvilgiu. Piktavalis parašo autorius arba igaliotinis gali apsimesti kitais igaliotiniais tam, kad sukurtu igaliotuosius parašus. Šiame straipsnyje, mes pateiksime mūsu atliktą Hsu et al. schemos analizę ir pasiūlysime naują slenkstini igaliotaji parašą, kuris neturi anksčiau nurodytų trūkumų. 